AGE SPECIFIC DEATH RATES FROM DIARRHOEA

\begin{tabular}{|l|c|c|}
\hline \multirow{2}{*}{$\begin{array}{c}\text { Place } \\
\text { \& year }\end{array}$} & \multicolumn{3}{|c|}{$\begin{array}{c}\text { specific death rates } \\
\text { per 100,000. }\end{array}$} \\
\cline { 2 - 3 } & $0-11$ months & $1-4$ years \\
\hline New York, 1900 & 5603 & 398.7 \\
\hline New York, 1961 & 45 & 2.4 \\
\hline $\begin{array}{l}\text { Punjab, 1959 } \\
\text { India }\end{array}$ & 3446 & 312 \\
\hline
\end{tabular}

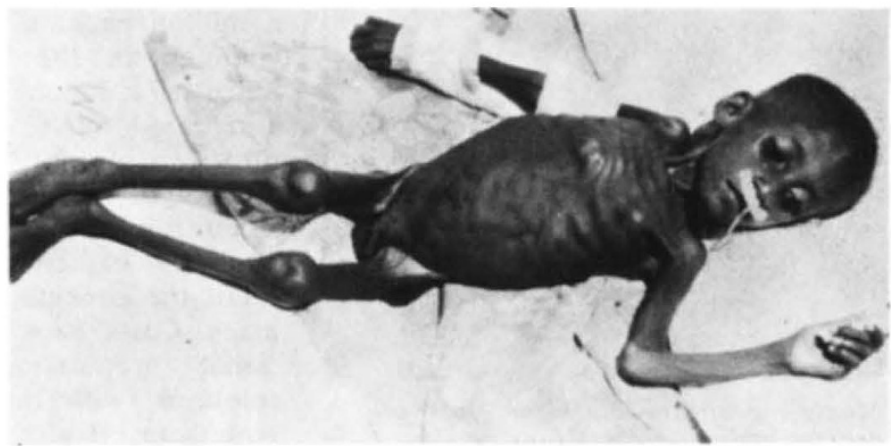

From Gordon et al: (1963)
Amer.J.Sci. 245: 345)

\title{
A cure for a killer-but how to deliver it?
}

\section{Millions of Third World children die of diarrhoea each year, although there is a simple cure available: oral rehydration. Discovered by scientists a decade ago, it has still not reached the people most in need. Anil Agarwal reports}

THE possibility of oral rehydration has provided Third World health authorities with a very valuable breakthrough in the treatment of diarrhoeal diseases. Loss of fiuids and electrolytic salts is the major complication in acute diarrhoeas, and if uncorrected, the continuing loss results in shock and eventually death. Until now rehydration has been possible only by administering fluids intravenously, as the small intestine loses its ability to absorb sodium during an attack of acute diarrhoea. Oral rehydration has now become feasible, however, with the observation that glucose absorption remains largely intact, and that sodium is carried along with it.

The application of this discoverylargely the result of work carried out at the Infectious Disease Hospital, Calcutta, the Cholera Research Laboratories, Dacca, Johns Hopkins University and the US Centre for Disease Control, Atlanta-now gives mothers the opportunity to treat their children suffering from diarrhoea themselves, by making a simple solution of glucose and electrolytic salts and feeding it to them.

\section{Millions of deaths}

Mothers no longer have to take their suffering children to health centres and hospitals tens of kilometres away, merely to seek the qualified nursing abilities required for intravenous treatment. For the majority of Third World mothers, who still do not have any reasonable access to health centres and hospitals, the necessity for intravenous treatment has really meant no treatment at all, and hence death for their children-a report from a research project situated in the relatively wellserved region of Punjab, India, recently pointed out that though medical orders to village health workers called for children with clinical signs of dehydration to be immediately referred to the physician at the health centre, many children still died at home of dehydration after they had been seen by the health worker. Many other children died without being seen at all. In this region, diarrhoeas were found to be responsible for $44 \%$ of all deaths amongst children less than three years of age. Considering that in many parts of the Third World, deaths amongst children constitute nearly half of all deaths in the population, the control of diarrhoeal diseases is of vital importance for the improvement of Third World health. It is estimated that there are some 500 million episodes of diarrhoea every year amongst children below the age of five. Experts differ, but it is widely accepted that these attacks result in the death of some 5-20 million children every year.

It is ironic that though diarrhoeal diseases have been reduced to a mere inconvenience in the developed countries, they still constitute the biggest cause of death and ill-health in the Third World. The primary cause of diarrhoeas is, of course, lack of clean water and generally insanitary conditions. But unfortunately these conditions are quite unlikely to change for at least another decade or two. WHO surveys show that as much as $78 \%$ of the Third World's rural population is still without clean community water supplies and about $85 \%$ is without adequate excreta disposal facilities.

Given these dismal conditions, oral rehydration has certainly opened up a glorious opportunity to take treatment, if not prevention, of diarrhoeal diseases to every doorstep in the Third World, however remote it might be, and control their worst consequences, dehydration and shock. In fact, oral rehydration can render an even more valuable service by helping to overcome the debilitation caused by the overwhelming majority of diarrhoeal attacks, which are mild and moderate, and do not lead to acute complications like dehydration. Together with mal- nutrition, these diarrhoeas combine to form a vicious, mutually reinforcing complex of disease and ill-health. Malnutrition increases susceptibility to diarrhoeal attacks and persistent diarrhoeas increase malnutrition. If a child somehow survives the combined influence of these two, then a minor disease like measles often proves sufficient to kill him. However, studies now show that a child regularly treated with oral fluids should be able to maintain relatively good health. Trials in the Philippines for seven months, and in Turkey for 16 , revealed that children who were given oral fluids had much higher monthly weight gains than those children who were left untreated: the mean weight gain of the non-treated children was signficantly below that of the average growth curve.

\section{Tests}

The first serious test of oral rehydration took place in 1971 when cholera epidemics were taking a heavy toll of millions of Bangladeshi refugees who had poured into India. Two or three physicians, assisted mainly by the illiterate family members of the patients, were able to treat with oral fluids 3,700 severely ill refugees in less than two months. It was a state of total confusion, with up to 200 new patients being admitted daily; even space on the mud floor was full of purging patients. But only 125 patients $(3.6 \%)$ diedand half of these before they could be given any fluids. By contrast, $9 \%$ of cholera cases in Italian hospitals died in 1973.

WHO and UNICEF are now spear-

Above: left, the death rate tells its own story. Right, the malnourishment of this child obscures diagnosis. The combination of symptoms is not rare in cases of diarrhoea, where repeated attacks worsen the condition. The majority of cases occur within the age group 6 to 18 months. 


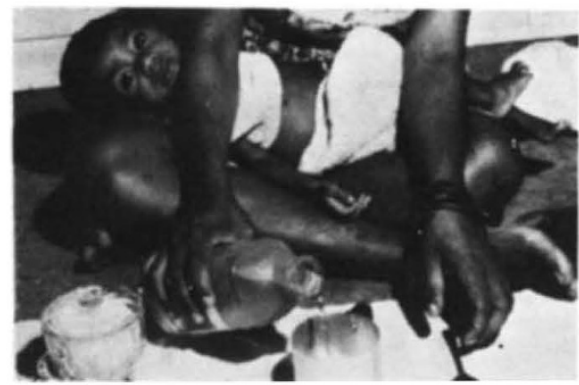

Measuring bottles and clean glassware are available in hospitals, as here in Papua New Guinea, but not in villages.

heading an international campaign to persuade Third World governments to introduce oral rehydration work in their primary health care activities. Numerous WHO-sponsored field studies are in progress, or have been completed in Egypt, El Salvador, Guatemala, India, Iran, the Lao People's Democratic Republic, Liberia, Nigeria, Turkey and the Philippines. Through these studies, WHO hopes it will be possible to convince local health administrators of the practicability and benefits of the new method. Many Third World hospitals have already adopted oral rehydration. The great majority of cholera patients at the Infectious Diseases Hospital in Calcutta, for instance, are now being treated in this way. The switchover from intravenous to oral fluids has meant a saving of about 50,000 dollars every year for the hospital in the cost of the fluids alone. More than a third of the beds in children's hospitals and wards in developing countries are usually occupied by diarrhoea cases receiving expensive antibiotics and intravenous fluids, thus putting a heavy load on their limited budgets. These units will greatly benefit from oral rehydration.

\section{Delivery}

Exactly how this new revolutionary therapy should be delivered to the Third World's million villages is, however, a matter of controversy amongst child health experts. WHO believes that the ideal course of action, in the interest of standardisation and quality control, is to prepare packets of oral rehydration salts in a standard mixture ( $3.5 \mathrm{~g}$ sodium chloride, $2.5 \mathrm{~g}$ sodium bicarbonate, $1.5 \mathrm{~g}$ potassium chloride and $20 \mathrm{~g}$ glucose) that would make a litre solution, and then sell these packets widely through commercial channels or distribute them through rural health centres. UNICEF is helping developing countries not only to distribute these packets but also to set up units to produce them. The packet approach is gaining acceptance. UNICEF has already distributed more than a million packets in developing countries, while Royal Drugs Ltd in Nepal has marketed over a quarter of a million packets through commercial channels in the Kathmandu Valley. Since 1974, Indonesia has been running a national rehydration campaign with provision of locally packaged glucoseelectrolyte mixture to its more than 2,000 rural health centres, and large numbers of commercial firms also retail the packets in the open market place. Costa Rica has instituted a national programme using packaged solutions, with the help of the PanAmerican Health Organisation. The Cholera Research Centre in India has launched a drive to popularise oral fluid therapy among doctors and the lay public. Under contract from the Indian Council of Medical Research, a reputable drug firm has been entrusted with the large-scale production of oral rehydration packets.

WHO's "appropriate technology for health" programme is simultaneously trying to find cheap and simple packaging methods, in the interest of the poorer consumers: packaging constitutes as much as $50-75 \%$ of the price of producing a pre-packaged mixture. Machine-packaging is one possibility, using a vacuum sealed aluminium packet, coated with a plastic membrane inside. This type of packaging would minimise the moisture content, thus increasing the shelf-life of the powder. The price of a 1-litre machine-packaged UNICEF packet is currently estimated at 6.5 US cents. Hand-packaging would be cheaper and would create more employment, though the shelf-life of the powder would be less. In many rural areas of the Third World there already exist small packaging workshops where household articles and some drugs are packaged into polythene bags, and such workshops would be ideal for the hand-packaging of glucose-electrolyte mixtures. The cost of such mixtures is estimated to be about 5 US cents. All the ingredients could be put together in one polythene packet, or, because hand-packaging retains some moisture, the salts could be packaged separately and then added to a packet containing glucose. The cost of the latter technique would be higher, but the shelflife would be increased.

The major advantage of prepackaged solutions is that mothers would get the quantity of each ingredient correctly measured. An excess of potassium salts can be toxic. Large excess quantities of sodium can cause hypernatraemia, a condition that affects the heart.

\section{Critics}

There are, however, several critics of the WHO-UNICEF approach. Their arguments are largely based on the grounds that this approach has not adequately taken into account the extent of poverty of a large percentage of the rural population and on the logistic difficulties of distributing centrally-produced packets to every village in the Third World. Packaging, which only adds to costs, can make even this simple and cheap oral rehydration therapy virtually prohibitive for millions of mothers. Distribution through commercial channels increases the price further by adding profits for manufacturers, distributors and retailers. Although these packets will eventually be required in hundreds of millions, manufacturers have not leaped into production, because, it seems, of the low profits at which they would have to sell them to reach the mass of the consumers. Distribution through health centres would also not reach the majority of the Third World rural population. In Indonesia, for instance, it has been estimated that distribution of packets through health centres has reduced mortality to less than $5 \%$ in dehydrated children, but only $10 \%$ of the total cases actually use health centres.

Another problem with the WHO standard 1-litre solution is that in most villages of the Third World mothers will not find it easy to measure a litre. Instead, every culture has some standard measure, based on a readily available container: in India, for instance, a $300 \mathrm{ml}$ beer bottle can be found almost everywhere. But packaging in smaller quantities will only increase the cost of packaging, and hence the total cost of treatment. Moreover, keeping a litre of aqueous solution

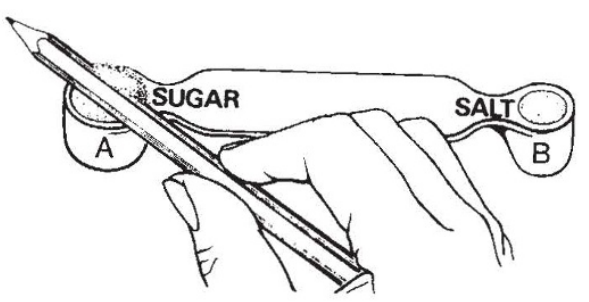

This simple measuring spoon was developed for home use in Indonesia.

containing glucose for more than a few hours is not advisable in unhygienic rural conditions, as it would provide an excellent culture for several pathogenic organisms. Instead, it would be better to make the solution every time it is needed.

\section{Mother the focus}

The alternative approach being suggested is to make the mother the focus of oral rehydration treatment-to enable her to make and administer the solutions herself. Jon Rohde, who is working with a Rockefeller Foundation project on oral rehydration in Indonesia, argues that dehydration is just a form of malnutrition: fluidelectrolyte malnutrition. The key role of the mother in the treatment of 
protein-calorie malnutrition is widely accepted. "Her role in replacing fluid and electrolytes is just as crucial," says Rohde, "perhaps more so considering the acute course of fluid-electrolyte malnutrition."

To enable the mother to treat her child herself, the WHO standard mixture can be considerably simplified. One problem is the availability of glucose, which has to be imported by most developing countries. Rural mothers will find it very difficult to obtain it. Glucose could therefore be replaced by the easily available white sugar (sucrose) which breaks down to glucose in the intestine. Sugar-salt solutions have indeed been tested by several researchers and have been found to be almost as effective as the WHO standard mixture in helping acute diarrhoea patients to recover. The simplicity of the sugar-salt solutions makes it possible for virtually every mother to become self-reliant. If nothing else is available, Dr William Cuttings of the London School of Hygiene and Tropical Medicine even recommends that mothers would do very well to use brown sugar or honey, which is available in many rural areas where beekeeping is a traditional practice. For potassium a mother could possibly squeeze in some orange or any other citrus fruit.

Researchers at the Institute of Child Health, Lagos, have found that poor mothers adopt the sugar-salt solution with great alacrity. All the
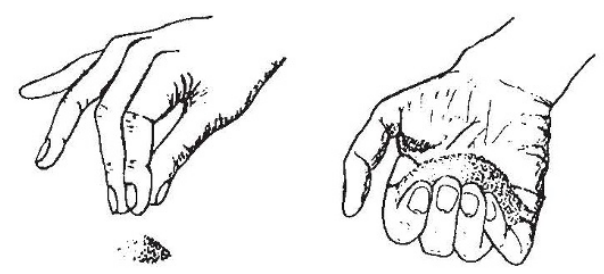

Fingers alone can measure quantities roughly. Left, $1.5 \mathrm{~g}$, right, $30 \mathrm{~g}$.

women attending the Institute's clinic are being taught oral rehydration therapy. In a recent survey, even women expecting their first child and others who had never used the service were able to describe the treatment. The survey however revealed that nearly half of the 217 women interviewed were planning to use more than four times the quantity of salt suggested to them (one-quarter teaspoon in a standard local beer bottle filled with water) and three cases were going to use sixteen times as much. Even vey educated Western mothers have the tendency to believe that if something is good, more of it is even better.

To solve the problem of accurate measurement, a special plastic spoon has been developed in Indonesia which has two separate indentations for measuring salt and sugar. This plastic spoon costs just 0.5 US cents. Such spoons are not only cheap, they could easily be made to suit local measures. Jon Rohde is using these spoons to teach children in primary schools and groups of mothers to make sugar-salt solutions. Dr Zafarullah Choudhury, a leading primary health care innovator from Bangladesh, is also teaching schoolchildren to make their own solutions. Because of its simplicity, Rohde strongly argues oral rehydration should be made everybody's business: "Teachers, agriculture extension workers, government employees, boy scouts, women's groups and even schoolchildren should be taught to mix rehydration fluids and use them."

\section{No global method}

Dr Christopher Lomax of the London-based Appropriate Health Resources and Technologies Action Group argues that "as in most 'appropriate technologies' it is difficult to recommend a single method to be used on a global scale, for each method will be viewed from a different perspective in different parts of the world".

Dr David Morley of the Institute of Child Health, London, who also strongly supports the use of spoons for measuring, believes that it would be best if national oral rehydration programmes were designed to operate at different levels of sophistication. At the level of the health centre, where even seriously dehydrated cases can be expected to turn up, both oral and intravenous therapy should be provided using the WHO standard mixture. But at the level of the individual mother, who has to treat most of the time mild and moderate diarrhoea cases, health authorities should promote the use of simple sugar-salt solutions made with the help of spoons.

WHO's Director-General, Halfdan Mahler, has in his speeches favoured the mother-based approach as best both in the long and short term. For WHO's new community-based approach to primary health care to succeed, Mahler has argued that a demystification of medical technology is needed. Oral rehydration, he believes, is an extremely good example where the responsibility of the community's health can be put into the hands of the community itself.

\section{Maintaining standards}

WHO's officials, however, who are very conscious of the organisation's reputation for medical standards, find it necessary to be cautious. WHO does not oppose any studies to test the efficacy of home-made sugar and salt solutions. But as all the major studies that have been published to date on the

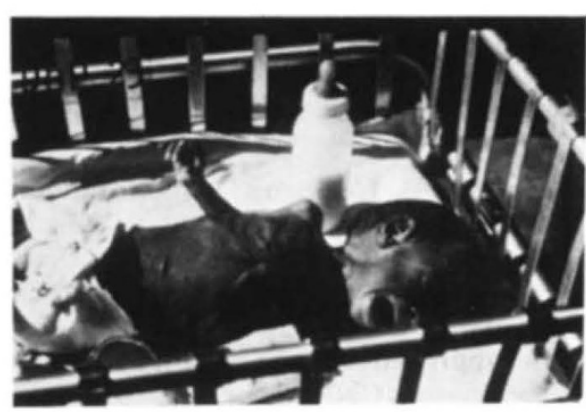

The trend to bottle-feeding among the urban poor increases risk. Poverty means the mixtures are over-dilute, leading to malnourishment, and sterilisation of the bottles is impossible.

efficacy of oral rehydration have used the complete formula, WHO is making all attempts to promote widespread distribution of packets containing the standard mixture. It is felt that with sufficient education by health auxiliaries the complete formula can be mixed by mothers at home. With recurrent use of this solution, less education would be needed, and therefore hopefully there will soon be a minimum need for homemade sugar-salt solutions.

Dr Richard Cash of Harvard University also opposes attempts to simplify the oral rehydration therapy too fast. He believes that the medical community should not be bypassed. It is possible to go to the people directly and teach them self-help, but local doctors too must be adequately convinced of the effectiveness of a therapy before it is widely promoted. Otherwise, they will only discourage people from using them.

Furthermore, Dr Cash argues, the real problems in reaching the people is not scientific-whether one solution should be used or another-but lack of political will. It has taken ten years for governments to wake up to the possibility of oral rehydration since it was first indicated by scientific research. Producing packets and selling them is a far simpler job than undertaking a massive programme to educate every mother about oral rehydration. Governments, which still have to pick up the courage to provide more than half their population with adequate health services, are more likely to adopt the easier alternative.

The debate about oral rehydration does show that it is often easier to generate new medical knowledge that meets the needs of the neglected, underserved poor of the world, than it is to find ways to enable them to benefit from that knowledge. The debate about the delivery of oral rehydration parallels virtually every issue that has been raised in the more general primary health care debate about delivering health services to the rural Third World. 